Research Article

\title{
Underwater Terrain-Aided Navigation Relocation Method in the Arctic
}

\author{
Yanji Liu $\mathbb{D}^{1},{ }^{1}$ Guichen Zhang $\mathbb{D}{ }^{1}$ and Chidong Che $\mathbb{D}^{2}$ \\ ${ }^{1}$ Merchant Marine College, Shanghai Maritime University, Shanghai 201306, China \\ ${ }^{2}$ State Key Laboratory of Ocean Engineering, Shanghai Jiao Tong University, Shanghai 200240, China
}

Correspondence should be addressed to Yanji Liu; liuyanji@tongji.edu.cn

Received 31 October 2020; Revised 3 December 2020; Accepted 13 December 2020; Published 29 December 2020

Academic Editor: Kai Wang

Copyright (c) 2020 Yanji Liu et al. This is an open access article distributed under the Creative Commons Attribution License, which permits unrestricted use, distribution, and reproduction in any medium, provided the original work is properly cited.

To solve the localization failure problem of terrain-aided navigation (TAN) system of the autonomous underwater vehicle (AUV) caused by large area of underwater flat terrain in the Arctic, a navigation system with relocation part is constructed to enhance the robustness of localization. The system uses particle filter to estimate the AUV's position and reduce the nonlinear noise disturbance, and the prior motion information is added to avoid the mismatching caused by the similar altitude of low-resolution map. Based on the estimate data and the measured altitude data, the normalized innovation square (NIS) is used to evaluate the differentiation of terrain sequence, and the differentiation is used as a judgment of whether the AUV is in the switch location. A simulation experiment is carried out on the $500 \mathrm{~m}$ resolution underwater map of the Arctic. The results show that adding the prior motion information can restrain the divergence of the estimator; NIS can accurately reflect the sharp change of terrain sequence. After the relocation process, the AUV can still maintain the positioning accuracy within $2 \mathrm{~km}$ after running $50 \mathrm{~km}$ in the area including flat and rough terrain. This research solves the problem of localization errors in the Arctic flat terrain in the system level and provides a solution for the application of underwater navigation in the Arctic.

\section{Introduction}

The Arctic region has a vast water area and rich natural resources. However, the ice and snow covered on the surface of the water have hindered the application of GPS in the Arctic underwater navigation, reduced the large-scale navigation ability of underwater vehicles in Arctic waters, and increased the difficulty of underwater information collection $[1,2]$. In addition, the limited energy carried by AUV limits the use of high-power navigation equipment. Although there are many research on the efficient utilization of batteries and controllers [3-6], low-power navigation mode is still needed for AUV continuous operating under a large area of Arctic ice. TAN uses the real-time altitude information to match the prior DEM (digital elevation map) and obtains the optimal calculated position through the optimization estimation method [7-9]. Since it is not necessary to deploy external auxiliary sensors to obtain global positioning reference posture, only a prior DEM and a low-power sensor which obtaining altitude are needed. With these advantages, TAN technology has been widely used [10-12].

TAN was first used in cruise missiles and has been widely used in underwater navigation [13, 14]. The early TAN system mainly used terrain contour matching (TERCOM) technology to obtain positioning information through altitude dataset matching. TERCOM technology is relatively mature, but it cannot effectively deal with process noise and observation noise $[15,16]$. Among the three common regression filtering methods, Kalman filter [17, 18], point mass filter $[19,20]$, particle Filter (PF) [21, 22], Kalman filter requires the observation to be Gaussian distribution; it is not suitable for the application in non-Gaussian parameter situation. The point mass filter can solve the expression of 
non-Gaussian observations, but it needs to calculate the whole posterior distribution space, which requires a large amount of calculation. Compared with point mass filter, the PF method has less computation and has a resampling mechanism to maintain the diversity of sampling and can deal with the ubiquitous nonlinear noise, which has been widely used [23-27]. Georgios [28] used the PF method to build the TAN system and carried out a long-range navigation accuracy test in the Southern Ocean. Based on the DEM with the resolution of $50 \mathrm{~m}$, the positioning accuracy of TAN is within $200 \mathrm{~m}$ in multiple tests when the average moving distance of AUV is greater than $100 \mathrm{~km}$, which can meet the needs of accurate positioning of underwater vehicles in a large-range operation.

The DEM of TAN and the underwater altitude detected by AUV both have noise disturbance, which will affect the positioning accuracy [29]. In extreme cases, when the noise disturbance is very large or when the terrain environment changes, it will lead to the failure of positioning. The commonly solutionis is to use the hypothesis-test method to calculate the confidence level of the state estimate HUGIN AUV uses the chi-square test method to estimate the confidence degree of the system and then makes a trade-off for the TAN estimation value [30].

At present, the resolution of the Arctic underwater DEM is only $500 \mathrm{~m}$ [31], and the low-resolution map has a large error with large noise, which affects the estimation of the location of AUV [32]. Salavasidis et al. [33] utilize the bilinear interpolation method to improve the resolution of the map, but it cannot eliminate the map noise. In addition, there are more flat terrain areas in the Arctic terrain, and the flat terrain with few features reduces the positioning ability of TAN, which leads to positioning failure. At present, there are many methods to improve the accuracy in a flat area; Aringnonsen and Hagen [34] utilize the pockmarks in the flat terrain as a sign to improve the positioning accuracy. Enns and Morrell [35] utilize the Viterbi algorithm to calculate the Bayesian dataset to obtain the most likely position of the present motion. Shandor and Stephen [36] adjusting the measurement variance to eliminate the over matching position and reducing the mismatching in the flat area. But more generally, in a large area of flat area, the deviation and matching value of altitude in all directions are similar.

When an AUV moves from a flat area to a rough terrain area, a robot kidnapping problem occurs. In the robot kidnapping problem, timing of triggering relocation is important [37]. In terrain aided navigation, it is necessary to accurately determine the switch location of flat terrain and rough terrain. Variance analysis can be used to distinguish the planeness of terrain, and the NIS method can produce more accurate results $[38,39]$. Houts et al. [40] used the NIS window to estimate the confidence of PF filtering results and judge the change of terrain according to the confidence. The results show that the NIS window filtering method can effectively reduce the impact of noise disturbance and can accurately positioning the abnormal. By improving the weight distribution of $\mathrm{PF}$ and optimizing the likelihood function, the robustness of the system can be enhanced and the influence of flat terrain can be reduced slightly, but the positioning error caused by large flat terrain still cannot be solved [41, 42].

To eliminate positioning errors in the TAN system caused by the large area of flat terrain in the Arctic, this paper proposes a TAN positioning framework with the relocation system. The NIS is used to construct the confidence evaluation item to accurately judge the moment when AUV enters the rough area. Based on the accumulated navigation data and the sampled altitude data in the flat area, the relocation function is constructed, and the relocation process ensures the convergence of the TAN system when it leaves the flat area and ensures the accuracy of TAN positioning in a large scale. In addition, in order to solve the problem of low-resolution map causing low-terrain differentiation in a large area, a priori motion information enhancement method is applied to improve the calculation of weight to improve the accuracy of positioning estimation.

\section{PF Position Estimation System}

We use PF to estimate the AUV location; the essence is to use $\mathrm{PF}$ to correct the position on the basis of dead reckoning. This paper focuses on the positioning ability of TAN system in the horizontal plane. Therefore, the simplified two-dimensional AUV kinematic model is constructed as the basis of dead reckoning. In this paper, the sequential importance sampling and resampling (SISR) algorithm is selected as the filter of the TAN system to estimate the position of AUV.

2.1. AUV Kinematics. As shown in Figure 1, it is a 3-DOF kinematic coordinate system of AUV in the horizontal plane [43]. [ $v$ and $w$ ] are the velocity and angular velocity of AUV relative to the body coordinate, $[x, y$, and $\theta]$ is the pose of AUV relative to the global coordinate, and $|v / w|$ is the radius of rotation of the AUV around its turning center. The kinematic model is shown in equation (1), in which the lower subscript $t$ represents the moment of motion estimation, and $\Delta t$ is the step interval time.

$$
\left(\begin{array}{c}
x_{t+1} \\
y_{t+1} \\
\theta_{t+1}
\end{array}\right)=\left(\begin{array}{c}
x_{t} \\
y_{t} \\
\theta_{t}
\end{array}\right)+\left(\begin{array}{c}
-\frac{v}{w} \sin \theta+\frac{v}{w} \sin (\theta+w \Delta t) \\
\frac{v}{w} \cos \theta-\frac{v}{w} \sin (\theta+w \Delta t) \\
w \Delta t
\end{array}\right)
$$




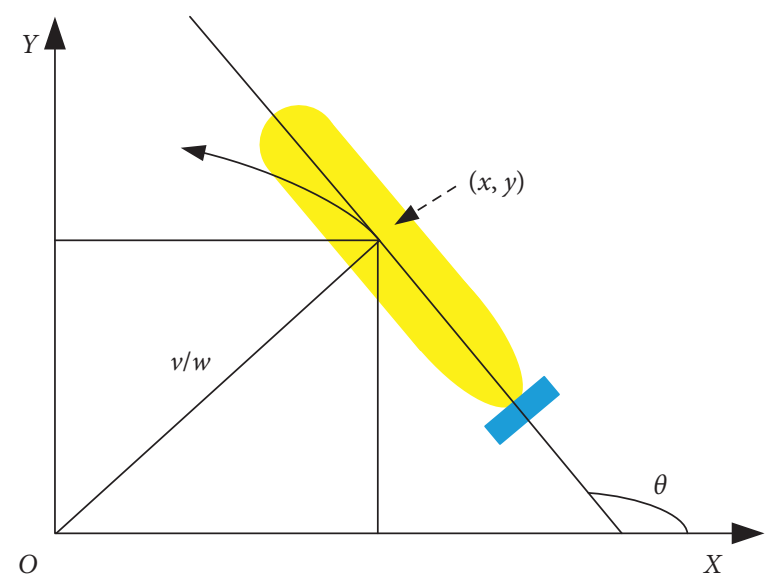

Figure 1: Three degrees of freedom of AUV kinematics.

The movement of AUV is mainly affected by two kinds of disturbance: one is the fluctuation of control input $[v, w]$ caused by the noise of airborne sensors such as inertial navigation and depth gauge; the other is caused by external factors such as ocean current and water density. Therefore, process noise can be divided into body noise and environmental noise, as equations (2) and (3) show, where $\sum_{p}$ is body noise covariance, $\sum_{e}$ is external noise covariance, the superscript $\wedge$ represents the state with noise, $N$ represents the normal distribution, $a_{i},(i=1,2,3,4)$ is constant coefficient, $\sigma_{x}^{2}$ is variance in $x$ direction, and $\sigma_{y}^{2}$ is variance in $y$ direction.

$$
\begin{aligned}
& \sum_{p}=\left(\begin{array}{l}
\widehat{v} \\
\widehat{w}
\end{array}\right)=\left(\begin{array}{l}
v \\
w
\end{array}\right)+\left(\begin{array}{c}
N\left(0, a_{1} v^{2}+a_{2} w^{2}\right) \\
N\left(0, a_{3} v^{2}+a_{4} w^{2}\right)
\end{array}\right), \\
& \sum_{e}=\left(\begin{array}{l}
\hat{x} \\
\hat{y}
\end{array}\right)=\left(\begin{array}{l}
x \\
y
\end{array}\right)+\left(\begin{array}{c}
N\left(0, \sigma_{x}^{2}\right) \\
N\left(0, \sigma_{y}^{2}\right)
\end{array}\right) .
\end{aligned}
$$

\subsection{Prior Information Enhanced PF(PIEPF) Algorithm}

2.2.1. SISR PF Algorithm. In this paper, the widely used SISR algorithm is used for positioning estimation [44]. There are many descriptions of SISR algorithm in the research literature. Here, the logic is briefly summarized. PF uses random distribution to approximate the probability density function of the system; by iterative calculation, the minimum variance estimation of the system state is obtained; PF can solve the state estimation problem of nonlinear system and is widely used. The SISR algorithm is shown in Table 1, where $x$ is state variable, $\omega$ is particle weights, $y$ is observation measurements, $N$ are the number of particles, the upper subscript $i$ is the particle index, and the lower subscript $k$ is the time dimension.where $p\left(y_{k} \mid x_{k}^{i}\right)$ is the likelihood function of observations and can be expressed as

$$
p\left(y_{k} \mid x_{k}^{i}\right)=\frac{1}{\sigma_{y} \sqrt{2 \pi}} e^{-1 / 2\left(y_{k}^{i}-y_{k} / \sigma_{y}\right)^{2}},
$$

where $\sigma_{y}$ is observation variance.
2.2.2. PIEPF. The low-resolution map results in similar terrain altitude in local area, and only using the observation value cannot effectively distinguish the difference of particles, which leads to the divergence of filtering. Combining the prior information of AUV motion and reasonably considering the influence of stable Arctic current, the particle weight including prior motion information is calculated as formula (4).

$$
\omega_{k}^{i}=\omega_{k-1}^{i} p\left(y_{k} \mid x_{k}^{i}\right) p\left(x_{k}^{i} \mid x_{k-1}^{i}\right),
$$

where $p\left(x_{k}^{i} \mid x_{k-1}^{i}\right)$ is the likelihood function of prior motion and can be expressed as

$$
p\left(x_{k}^{i} \mid x_{k-1}^{i}\right)=\frac{1}{\sigma_{x} \sqrt{2 \pi}} e^{-1 / 2\left(\operatorname{Dis}_{k}^{i} / \sigma_{x}\right)^{2}}
$$

where $\mathrm{Dis}_{k}^{i}$ is the Euclidean distance between each particle and the prior predicted position.

\section{Relocation System}

There are a large number of flat terrain areas without terrain features of the Arctic, which will lead to the divergence of TAN positioning. Although scholars have proposed a variety of methods to improve the positioning accuracy in the flat area, the improvement is limited, and it is not suitable for the Arctic with large flat terrain. In this paper, a TAN system with relocation item is designed. The system structure is shown in Figure 2. When the AUV moves from the flat area to the rough area and crosses the terrain junction point, the relocation flag is triggered.

In the process of relocation detection, the key item is to accurately determine the running area of AUV. However, the sensor and the prior DEM have large noise. Although the direct use of the measured altitude for comparative judgment is simple, it cannot eliminate the influence of noise, which is easy to cause misjudgment and positioning failure. NIS can evaluate the credibility of the measured values and reduce noise disturbance. When AUV operates in rough areas with rich terrain features or flat areas with less terrain 
TABLE 1: Algorithm of SISR PF.
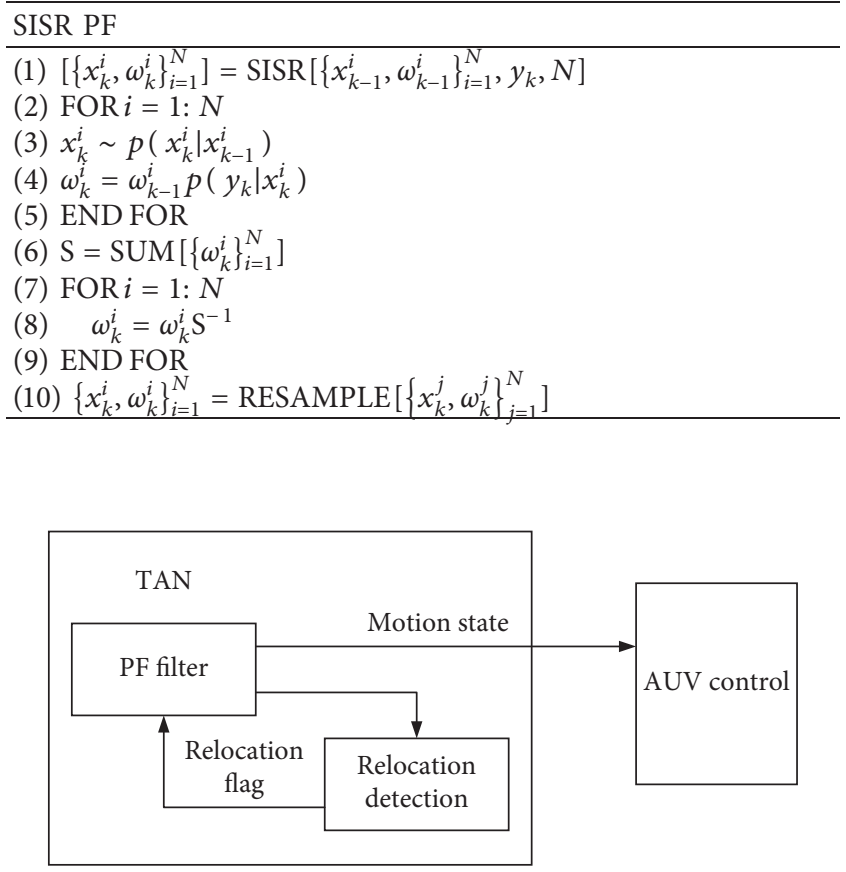

Figure 2: TAN system.

features, the measured values are close to the predicted values, and the NIS values are smaller. However, when the AUV enters the rough area from the flat area, the wrong estimated position will lead to a large difference between the measured value and the estimated value, resulting in a larger NIS value. In this paper, the NIS is used to construct a filtering window to estimate the confidence of observation; when NIS is greater than the threshold value, it indicates that the AUV enters the rough terrain from the flat terrain, which leads to the relocation process.

3.1. PF NIS. Innovation $\tilde{y}$ represents the difference between the measurements and the expected value as follows:

$$
\tilde{y}_{k}=y_{k}-\mu_{k}
$$

where $y$ is measurements, $\mu$ is expected value, low subscript $k$ is the moment, and the expected value is the weighted average of altitude.

$$
\mu_{k}=\sum_{m=1}^{M} \omega_{k}^{m} \widehat{y}_{k}^{m}
$$

where $\hat{y}$ is the observations of particles and $m$ is particle index.

Then, the variance of innovation $S$ is

$$
S_{k}=R_{k}+\sum_{m=1}^{M} \omega_{k}^{m}\left(\hat{y}_{k}^{m}-\mu_{k}\right)\left(\hat{y}_{k}^{m}-\mu_{k}\right)^{T},
$$

where $R$ is observation variance.

The NIS can be expressed as

$$
\mathrm{NIS}_{k}=\tilde{y}_{k}^{T} S_{k}^{-1} \tilde{y}_{k}
$$

Different from variance, NIS is a dimensionless number, which represents the consistency between the distribution of new observations and the distribution of observations. The variance of NIS conforms to the chisquare distribution, so the reliability of the measurements can be judged. If the NIS value is relatively large, the measured value does not fall into a reasonable distribution range, which indicates that there is a big difference between the measurements and the predicted value. In order to further reduce the influence of abnormal measurements, the average value of NIS in a period of time is used as the evaluation basis,

$$
\mathrm{NIS}_{k}=\frac{1}{L} \sum_{i=K-L+1}^{K} \mathrm{NIS}_{i}
$$

where $L$ is time length.

3.2. Relocation Range. In order to illustrate the relocation ability of the TAN system, a simple sampling logic is used to realize the sampling after relocation. The method is to accumulate AUV running distance when the NIS value is credible. When the NIS value exceeds the threshold value, the accumulated distance is used as the basis of relocation range. Search for a similar sampling altitude within the relocation range to realize the sampling in the observation value. Thelogical is as follows:

Step 1: If the NIS value is less than the threshold, the AUV motion distance Dis is accumulated

Step 2: When the NIS value is abnormal, 1.5.Dis is set as the resampling radius and the present altitude value $\mathrm{Hm}_{k}$ is recorded

Step 3: Within the sampling range, num particles are uniformly distributed

Step 4: Calculating particles altitude $H s_{k}^{i}$, when the difference between $H s_{k}^{i}$ and $H m_{k}$ is within the range of observation noise, the particle is preserved

After the above steps, resampling particles can be filtered out. After PF iteration, the particles in the wrong position will disperse rapidly, and the particles in the correct position can achieve good tracking. 


\section{Simulation}

In this paper, simulation is used to verify the effectiveness of the proposed relocation logic. Based on the Arctic DEM of $500 \mathrm{~m}$ resolution, a local area close to Alaska in the United States is selected for the simulation study. The area contains rough terrain and flat terrain, which is convenient for comparative analysis.

4.1. Map Parameters. The map with longitude range $\left(-180^{\circ}\right.$ and $\left.-168^{\circ}\right)$ and latitude range $\left(75^{\circ}\right.$ and $\left.79^{\circ}\right)$ is selected as the research area, as shown in Figure 3(a). In order to facilitate the analysis, the latitude and longitude map is transformed into the Cartesian coordinate system through scale conversion. The $3 \mathrm{D}$ terrain map is shown in Figure 3(b). The length, width, and height of the converted map are (800, 250, and -2.1$) \mathrm{km}$, and the origin point of the map corresponds to longitude $-180^{\circ}$ and latitude $75^{\circ}$.

In this paper, the bilinear interpolation is used to refine the map altitude. The bilinear method is shown in the following equation:

$$
\begin{aligned}
f(x, y)= & \frac{f\left(Q_{11}\right)}{500^{2}}\left(x_{2}-x\right)\left(y_{2}-y\right)+\frac{f\left(Q_{21}\right)}{500^{2}}\left(x-x_{1}\right)\left(y_{2}-y\right) \\
& +\frac{f\left(Q_{12}\right)}{500^{2}}\left(x_{2}-x\right)\left(y-y_{1}\right)+\frac{f\left(Q_{22}\right)}{500^{2}}\left(x-x_{1}\right)\left(y-y_{1}\right),
\end{aligned}
$$

where $f(\cdot)$ is the altitude function, $[x, y$, and $Q]$ is coordinate points, and the cross combination of $\left[x_{1}\right.$ and $\left.x_{2}\right]$ and $\left[y_{1}\right.$ and $\left.y_{2}\right]$ can form four corner coordinates $\left[Q_{11}, Q_{12}, Q_{21}\right.$, and $\left.Q_{22}\right]$ around the point to be solved.

4.2. TAN Parameters. The parameter setting of the TAN system is shown in Table 2. In order to eliminate the disturbance of vertical motion, the AUV is set to run at a depth of $0 \mathrm{~m}$, and in this situation, the observation noise is not a function of altitude but noise with a standard deviation of $5 \mathrm{~m}$. The numerical value conforms to the characteristics of the acoustic altimeter. In order to fully explore the performance of the system, a current disturbance in $Y$ direction of $0.1 \mathrm{~m} / \mathrm{s}$ is added.

4.3. Results and Analyses. Figure 4 shows the positioning results of the TAN system in rough and flat terrain areas, respectively. The running time of each movement segment is set as $28 \mathrm{~h}$, the running distance is $50 \mathrm{~km}$, and the movement direction is $180^{\circ}$. It can be seen that although there is the current disturbance in $Y$ direction, due to the rich terrain features, it has a good positioning results in the rough area. The estimated position calculated by TAN is very close to the reference path, and the average positioning error is within
$1 \mathrm{~km}$. In the flat terrain area, because of the lack of features, the TAN system cannot be accurately positioning, the movement path is random, and the positioning error is also divergent.

Figure 5 shows the comparison of path tracking error between PIEPF and ordinary PF. It can be seen that the overall tracking error of PIEPF is small and can effectively track the path. The PF method accurately tracks a certain distance and then diverges, which is caused by the similarity altitude of the low-resolution terrain. PIEPF method can track in the right direction with the aid of prior motion information.

Figure 6 shows the NIS values of TAN in different regions. It can be seen that the NIS values in the rough terrain area and flat terrain area are both within a $98 \%$ confidence interval. In the rough area, because of the accurate positioning, the observed altitude value is close to the predicted one, so the NIS value is small. In the flat terrain area, although it cannot be accurately located, because the altitude of the flat terrain area is close to the observation value, the difference is small, so the NIS value is small.

Figure 7 shows the positioning situation of AUV when moving from the flat area to rough area. When AUV is running in the flat area, due to lack of recognizable features, positioning fails, but the NIS value is kept within the credible range. When the AUV crosses the critical area and moves into the rough terrain area, there is a big deviation between the current measured altitude and the prior estimated one, which causes NIS anomaly. As can be seen from Figure 7(b), the NIS value increases rapidly and exceeds the trusted range.

Figure 8 shows the distribution of resampled particles and subsequent tracking and positioning. As shown in Figure 8(a), when the NIS value is abnormal, the cumulative distance of AUV is about $41 \mathrm{~km}$, and the sampling radius is $1.5 \times 41=62 \mathrm{~km}$. Within the sampling radius of $62 \mathrm{~km}$, 10,000 points are evenly sampled and 369 sampling points are finally accepted. Among them, 3 points are near the correct position and converge to the correct trajectory after $\mathrm{PF}$ iteration. Particles in other places diverge rapidly. Figure 8(b) shows the complete path, AUV loss in flat area, and when it reaches the rough area, it triggers relocation. After resampling, the AUV can return to the correct position.

\section{Results}

In this paper, we consider the characteristics of flat terrain and rough terrain in the Arctic region and use the NIS to trigger relocation, and the positioning failure caused by flat terrain is solved by relocation. The results show that the NIS value is sensitive to the area switching, which can accurately reflect the sharp change of terrain and can be used as a reliable judgment of the terrain area switching. In 


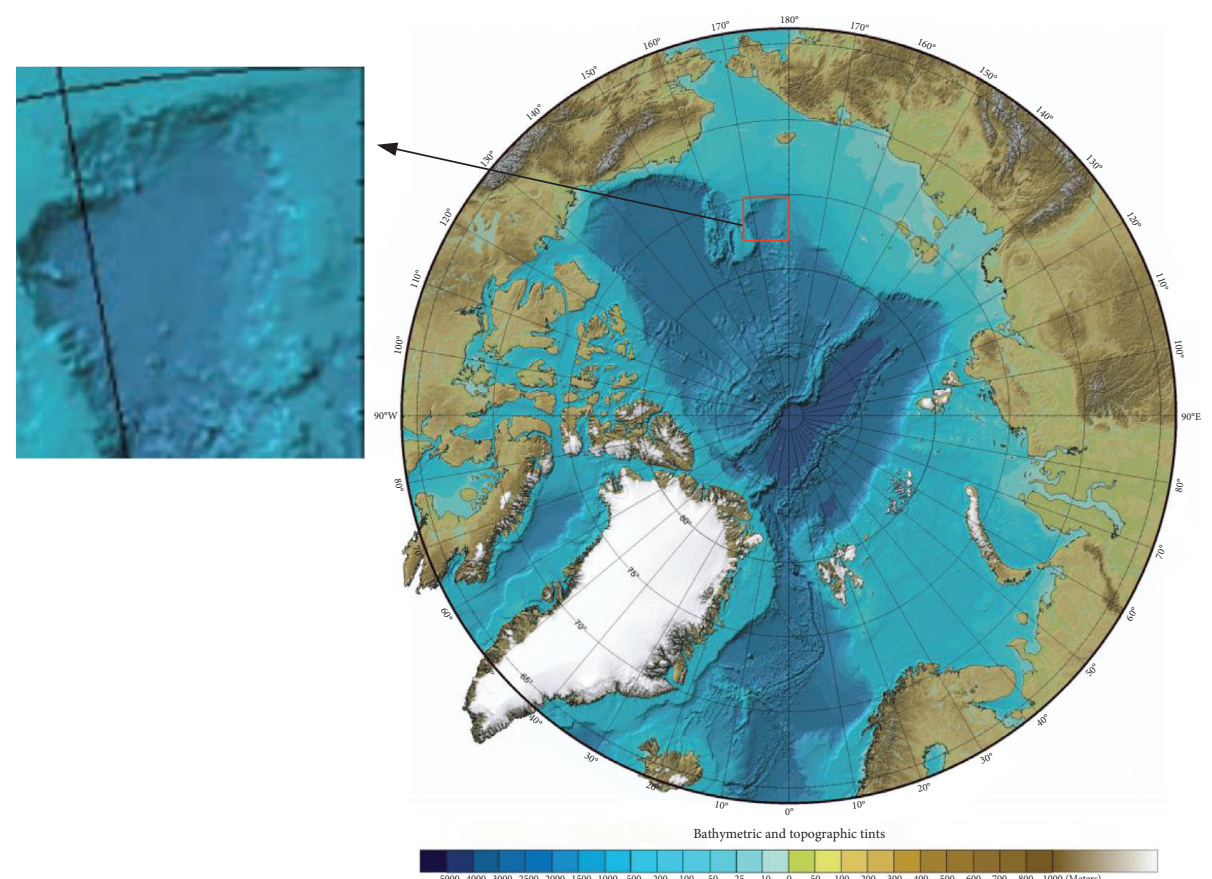

(a)

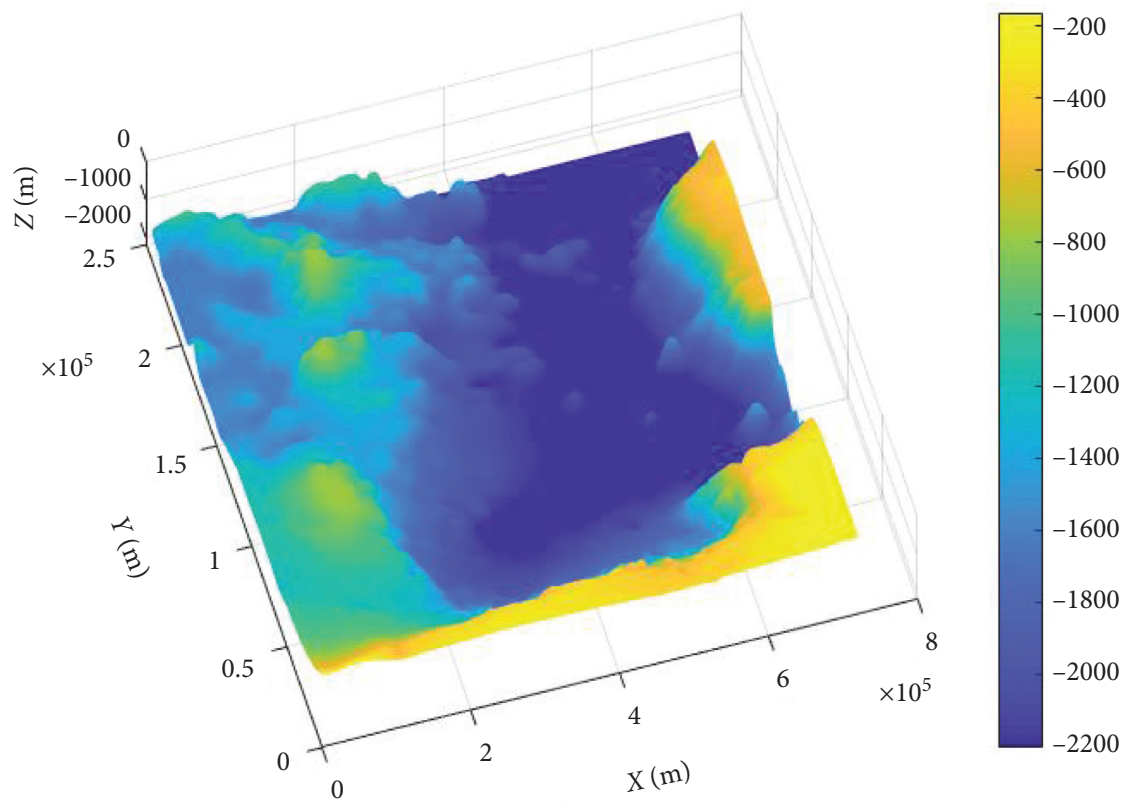

(b)

FIgUre 3: The terrain map of the research. (a) The selected area. (b) 3D DEM.

TABLE 2: Simulation parameters.

\begin{tabular}{lc}
\hline Parameters & Value \\
\hline AUV velocity & $0.5 \mathrm{~m} / \mathrm{s}$ \\
Map resolution & $500 \mathrm{~m}$ \\
Running depth & $0 \mathrm{~m}$ \\
PF update frequency & $1 \mathrm{~Hz}$ \\
Current disturbance & $(0,0.1 \mathrm{~m} / \mathrm{s})$ \\
$(d x, d y)$ & \\
\hline
\end{tabular}


TABLE 2: Continued.

\begin{tabular}{lc}
\hline Parameters & Value \\
\hline Body noise $\left(a_{1}, a_{2}, a_{3}, a_{4}\right)$ & $(0.1,0.1,0.001,0.1)$ \\
Process noise & $6 \mathrm{~m}$ \\
Measurement noise & $5 \mathrm{~m}$ \\
Particle numbers & 1000 \\
Initial attitude & $\left(200 \mathrm{~km}, 100 \mathrm{~km}, 180^{\circ}\right)($ rough area $),\left(500 \mathrm{~km}, 100 \mathrm{~km}, 180^{\circ}\right)($ flat area $)$, and $\left(290 \mathrm{~km}, 85 \mathrm{~km}, 180^{\circ}\right)($ flat-rough \\
NIS threshold THD & area $)$ \\
NIS time window $L$ & $5.41(98 \%$ confidence $)$ \\
\end{tabular}

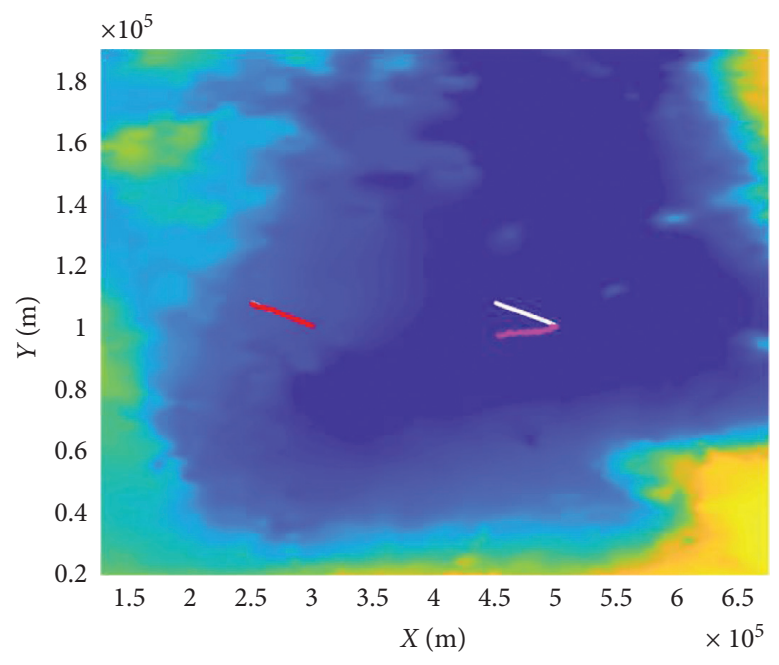

(a)

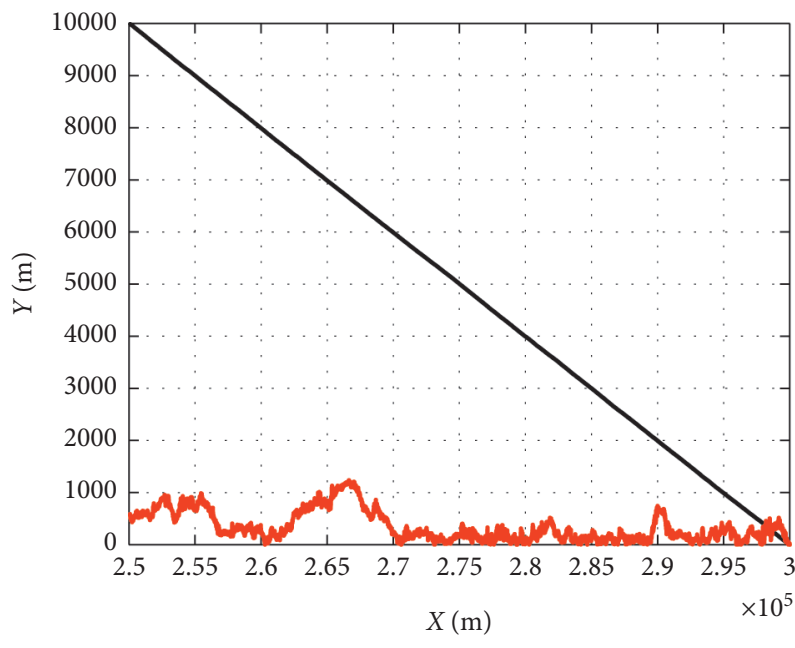

- Dead reckoning error

- PF tracking error in rough area

(b)

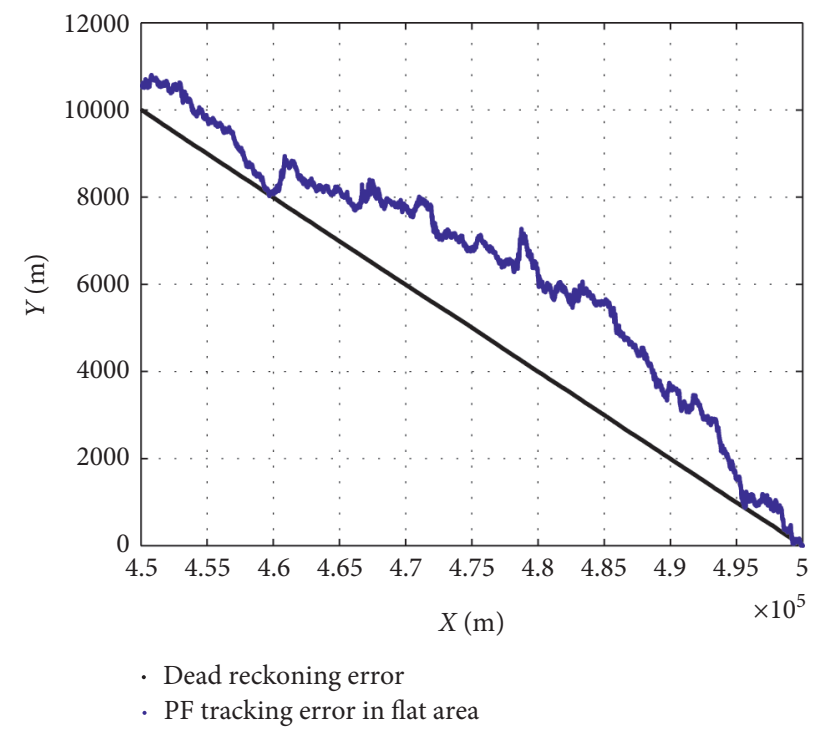

(c)

FIgURE 4: The localization results of TAN system in different terrain area. (a) TAN positioning path (the white path is the reference path, the red path is the positioning value in the rough area, and the pink path is the positioning value in the flat area). (b) Positioning error in rough area. (c) Positioning error in flat area. 


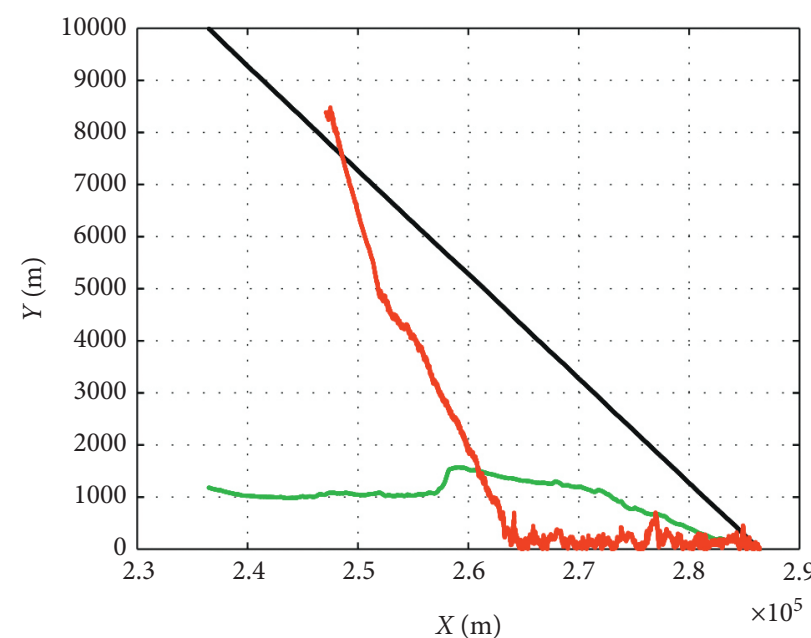

- Dead reckoning error
- PIEPF error
- PF error

FIgure 5: Tracking error of PIEPF and PF.

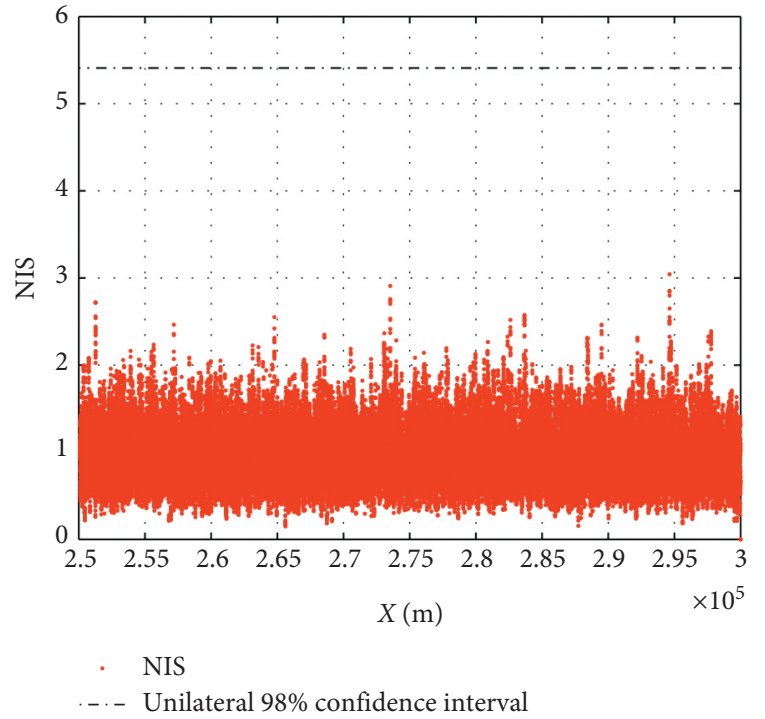

(a)

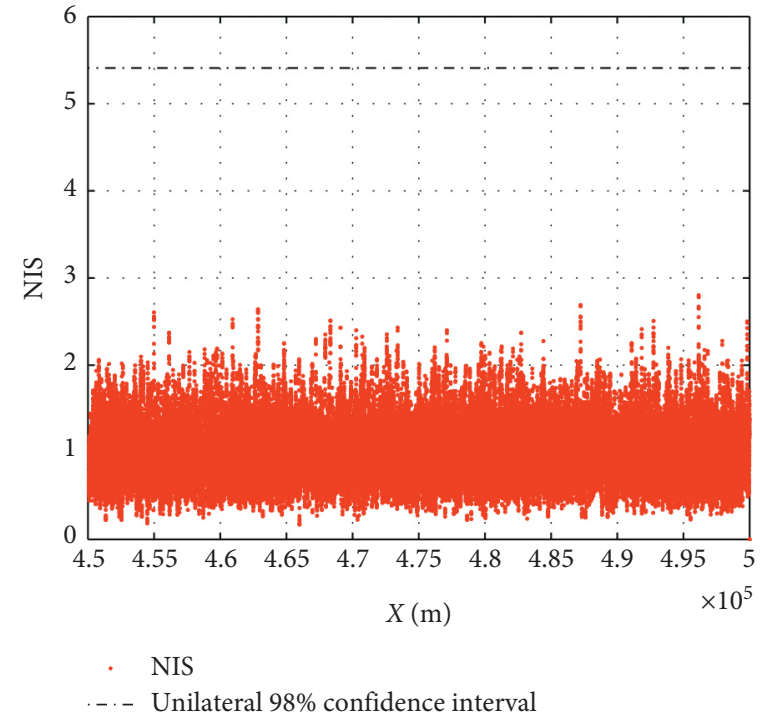

(b)

FIgURE 6: NIS values in different terrain area. (a) In rough area. (b) In flat area.

addition, on the basis of the PF framework, adding prior motion information can effectively avoid the divergence of filter, and the proposed method can be applied to underwater terrain aided navigation in the Arctic with a resolution of $500 \mathrm{~m}$.
The following research will further explore the NIS value characteristics under the condition of large map interference, improve the resampling distribution function, and improve the performance of the TAN system. 


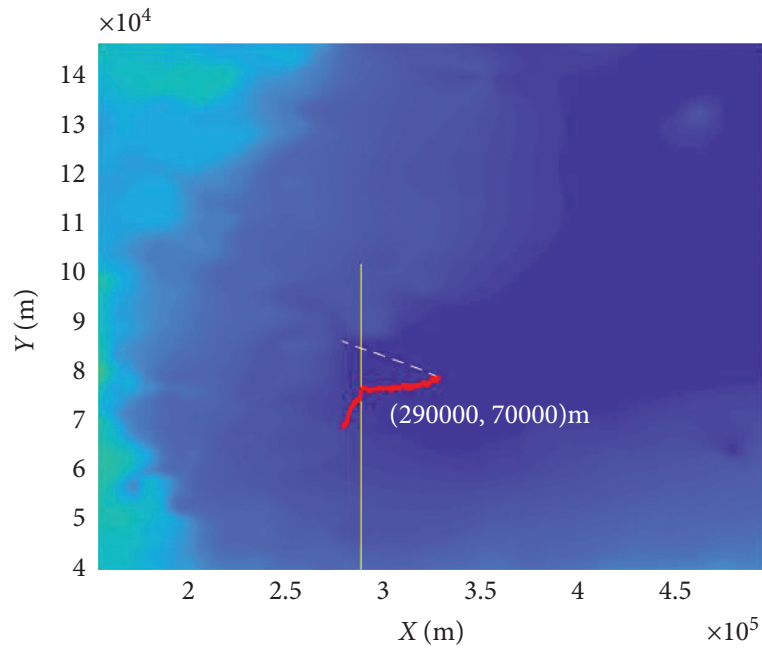

(a)

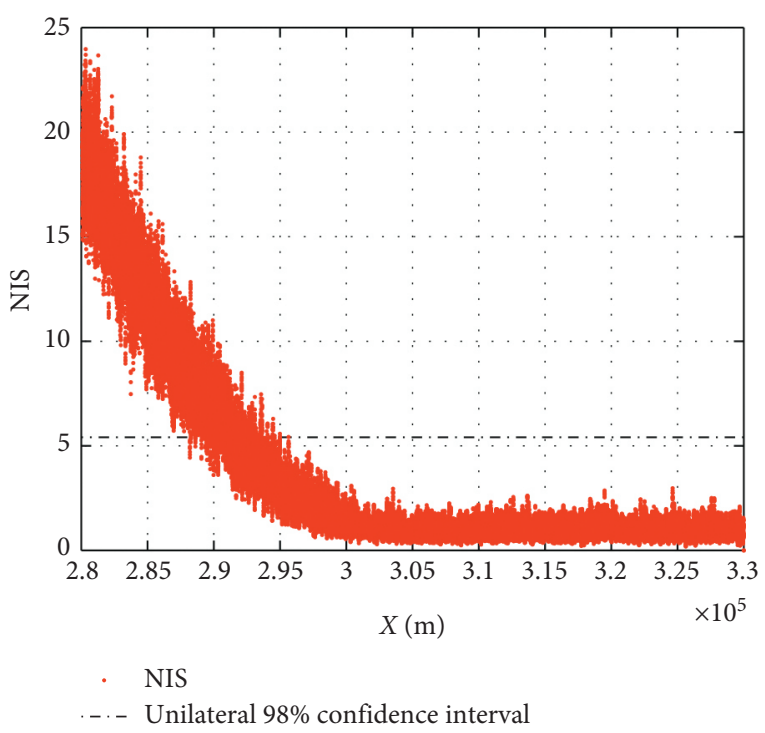

(b)

FIGURE 7: NIS outliers. (a) Movement path (the white dotted line represents the reference path, the red path is the actual positioning path, the intersection point of the yellow vertical line, and the red path is the critical position when entering the rough terrain). (b) NIS value.

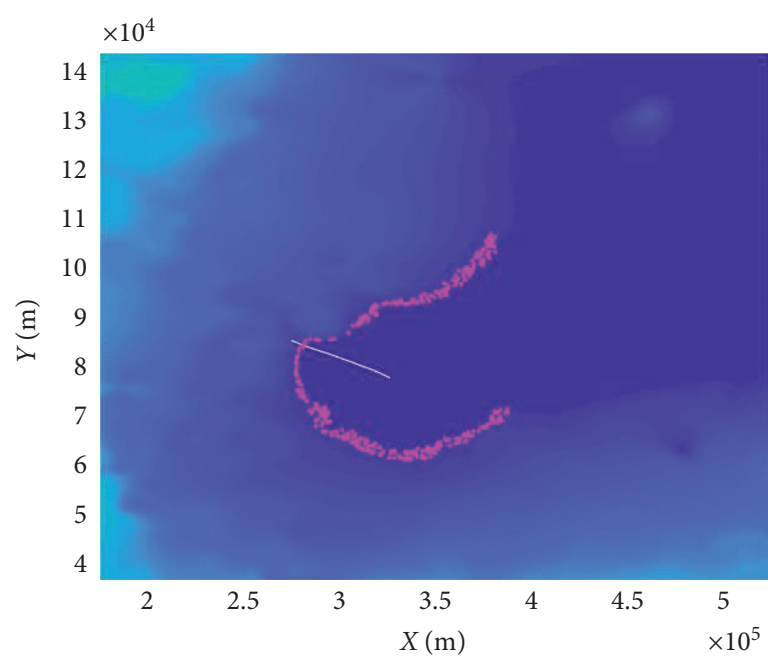

(a)

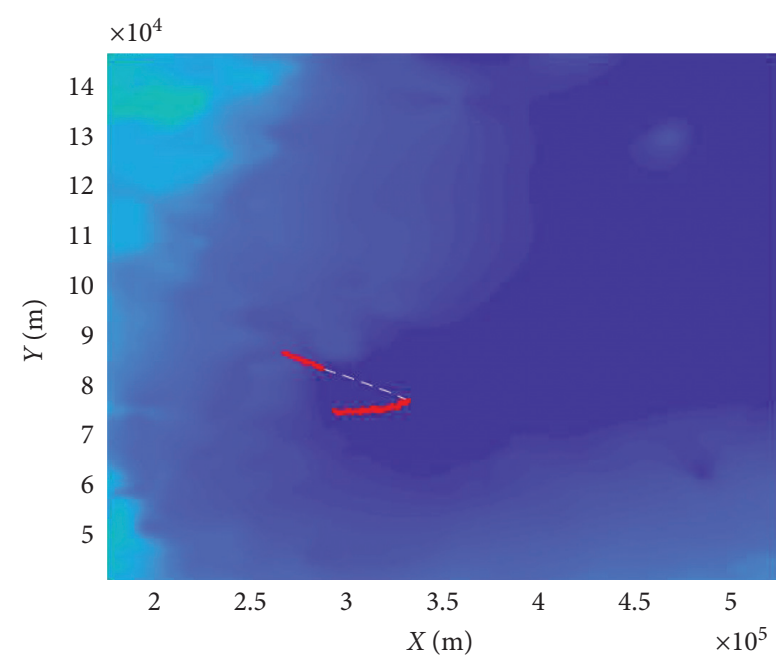

(b)

FIgURE 8: The tracking path of TAN system. (a) Resampling particles. (b) Tracking path.

\section{Data Availability}

The data used in this study are available from the corresponding author upon request.

\section{Conflicts of Interest}

The authors declare that they have no conflicts of interest.

\section{Acknowledgments}

This work was supported by the NSFC Projects of China under Grant no. 51779136 and Deep Blue Projects fund of
Shanghai Jiao Tong University under Grant no. SL2020MS002.

\section{References}

[1] L. Ruotsalainen, V. Renaudin, and L. Pei, "Toward autonomous driving in Arctic areas," IEEE Intelligent Transportation Systems Magazine, vol. 12, no. 3, pp. 10-24, 2020.

[2] Q. Zhang, Z. Chen, Y. Cui et al., "A refined metric for multiGNSS constellation availability assessment in polar regions," Advances in Space Research, vol. 66, no. 3, pp. 655-670, 2020.

[3] K. Wang, W. L. Wang, L. C. Wang et al., "An improved SOC control strategy for electric vehicle hybrid energy storage systems," Energies, vol. 13, no. 20, pp. 1-13, 2020. 
[4] K. Wang, F. Xiao, J. B. Pang et al., "State of charge (SOC) estimation of Lithium-ion battery based on adaptive square root unscented kalman filter," International Journal of Electrochemical Science, vol. 15, no. 9, pp. 9499-9516, 2020.

[5] K. Wang, L. Li, Y. Lan, P. Dong, and G. Xia, "Application research of chaotic carrier frequency modulation technology in Two-Stage matrix converter," Mathematical Problems in Engineering, vol. 2019, Article ID 2614327, 8 pages, 2019.

[6] G. Xia, Y. Huang, F. Li et al., "A thermally flexible and multisite tactile sensor for remote $3 \mathrm{D}$ dynamic sensing imaging," Frontiers of Chemical Science and Engineering, vol. 14, no. 6, pp. 1039-1051, 2020.

[7] M. Jose and M. Anibal, "Survey on advances on terrain based navigation for autonomous underwater vehicles," Ocean Engineering, vol. 139, pp. 250-264, 2017.

[8] Z. Song, H. Bian, and A. Zielinski, "Application of acoustic image processing in underwater terrain aided navigation," Ocean Engineering, vol. 121, pp. 279-290, 2016.

[9] D. Peng, T. Zhou, C. Xu, W. Zhang, and J. Shen, "Marginalized point mass filter with estimating tidal depth bias for underwater terrain-aided navigation," Journal of Sensors, vol. 2019, Article ID 7340130, 15 pages, 2019.

[10] E. J. Andrew and M. James, "Overview of terrain relative navigation approaches for precise lunar landing," in Proceedings of the IEEE Aerospace Conference Proceedings, Big Sky, MT, USA, March 2008.

[11] G. Salavasidis, A. Munafo, D. Fenucci et al., "Ultra-endurance AUVs: energy requirements and terrain-aidednavigation," in Autonomous Underwater Vehicles: Design and Practice, The Institution of Engineering and Technology, pp. 145-189, SciTech Publishing, Stevenage, UK, 2020.

[12] M. Christian, S. Bernard, B. Alexandre et al., "Terrain-aided navigation with an atomic gravimeter," in Proceedings of the 2019 22th International Conference on Information Fusion (FUSION), Ottawa, Canada, July 2019.

[13] I. Nygren, "Robust and efficient terrain navigation of underwater vehicles," in Proceedings of the IEEE/ION Position, Location and Navigation Symposium, pp. 923-932, Monterey, CA, USA, May 2008.

[14] S. Carreno, P. Wilson, P. Ridao et al., "A survey on terrain based navigation for auvs," in Proceedings of the IEEE OCEANS, Seattle, WA, USA, September 2010.

[15] B. I. Korelitz and S. C Sommers, "Responses to drug therapy in ulcerative colitis. Evaluation by rectal biopsy and histopathological changes," The American Journal of Gastroenterology, vol. 64, no. 5, pp. 365-370, 1975.

[16] L. Zhao, N. Gao, B. Q. Nan et al., "A novel Terrain-aided Navigation algorithm combined with the TERCOM algorithm and particle filter," IEEE Sensors Journal, vol. 15, no. 2, pp. 1124-1131, 2015.

[17] L. Hostetler and R. Andreas, "Nonlinear Kalman Filtering techniques for terrain-aided navigation," IEEE Transactions on Automatic Control, vol. 28, no. 3, pp. 315-323, 1983.

[18] L. M. Downes, T. J. Steiner, and J. P. How, "Lunar terrain relative navigation using a convolutional neural network for visual crater detection," in Proceedings of the 2020 American Control Conference, pp. 4448-4453, Denver, CO, USA, July 2020.

[19] D. Meduna, Terrain relative navigation for sensor-limited systems with application to underwater vehicles, Stanford Univeristy, Stanford, CA, USA, 2011.

[20] Y.-g. Park and C. G. Park, "Two-Stage point mass filter on terrain referenced navigation for state augmentation," Journal of Guidance, Control, and Dynamics, vol. 43, no. 5, pp. 1003-1008, 2020.

[21] K. B. Anonsen and O. Hallingstad, "Terrain aided underwater navigation using point mass and particle filters," in Proceedings of the IEEE/ION Position, Location, and Navigation Symposium, Coronado, CA, USA, April 2006.

[22] A. Bachmann and S. B. Williams, "Terrain aided underwater navigation-a deeper insight into generic monte carlo localization," in Proceedings of the Australasian Conference on Robotics and Automation, Brisbane, Australia, 2003.

[23] J. Melo and A. Matos, "On the use of particle filters for terrain based navigation of sensor-limited AUVs," in Proceedings of the MTS/IEEE Oceans'13 Conference, Bergen, Norway, June 2013.

[24] R. P. Wang, Y. S. Chen, Y. Li et al., "High-precision initialization and acceleration of particle filter convergence to improve the accuracy and stability of terrain aided navigation," ISA Transactions, 2020, In press.

[25] C. Zhang, C. Guo, and D. Zhang, "Ship navigation via GPS/ IMU/LOG integration using adaptive fission particle filter," Ocean Engineering, vol. 156, pp. 435-445, 2018.

[26] G. G. Rigatos, "Nonlinear kalman filters and particle filters for integrated navigation of unmanned aerial vehicles," Robotics and Autonomous Systems, vol. 60, no. 7, pp. 978-995, 2012.

[27] F. C. Teixeira, J. Quintas, and A. Pascoal, "AUV terrain-aided navigation using a Doppler velocity logger," Annual Reviews in Control, vol. 42, pp. 166-176, 2016.

[28] S. Georgios, M. Andrea, A. H. Catherine et al., "Terrain-aided navigation for long-endurance and deep-rated autonomous underwater vehicles," Journal of Field Robotics, vol. 36, no. 2, pp. 447-474, 2019.

[29] J. Lee, C. Sung, and S. Nam, "CRLB analysis for a robust TRN based on a combination of RNN and PF," International Journal of Aeronautical and Space Sciences, vol. 21, no. 1, pp. 265-276, 2020.

[30] B. A. Kjetil and K. H. Ove, "Recent developments in the HUGIN AUV terrain navigation system," in Proceedings of the OCEANS'11 MTS/IEEE KONA, Waikoloa, Hawaii, USA, September 2011.

[31] M. Jakobsson, L. Mayer, B. Coakley et al., "The international bathymetric chart of the arctic ocean (ibcao) version 3.0," Geophysical Research Letters, vol. 39, no. 12, pp. 1-6, 2012.

[32] D. Meduna, S. M. Rock, and R. McEwen, "Auv terrain relative navigation using coarse maps," in Proceedings of the Unmanned Untethered Submersible Technology Conference, Durham, NH, USA, August 2009.

[33] G. Salavasidis, A. Munafò, C. A. Harris, S. D. McPhail, E. Rogers, and A. B. Phillips, "Towards arctic AUV navigation," IFAC-PapersOnLine, vol. 51, no. 29, pp. 287-292, 2018.

[34] K. B. Aringnonsen and O. K. Hagen, "Terrain aided underwater navigation using pockmarks," in Proceedings of the OCEANS 2009, Biloxi, MS, USA, October 2009.

[35] R. Enns and D. Morrell, "Terrain-aided navigation using the viterbi algorithm," Journal of Guidance, Control, and Dynamics, vol. 18, no. 6, pp. 1444-1449, 1995.

[36] D. Shandor and R. Stephen, "Improving robustness of terrainrelative navigation for AUVs in regions with flat terrain," in Proceedings of the IEEE/OES Autonomous Underwater Vehicles(AUV), Southampton, UK, September 2012.

[37] I. Bukhori and Z. H. Zool, "Detection of kidnapped robot problem in monte carlo localization based on the natural displacement of the robot," International Jorunal of Advanced Robotic Systems, vol. 14, no. 4, 2017. 
[38] L. Cai, B. Boyacloglu, S. E. Webster et al., "Towards autotuning of kalman filters for underwater gliders based on consistency metrics," in Proceedings of the OCEANS 2019, Seattle, WA, USA, October 2019.

[39] A. J. Dean, J. W. Langelaan, and S. N. Brennan, "Improvements in terrain-based road vehicle localization by initializing an unscented kalman filter using particle filters," in Proceedings of the 2010 American Control Conference, pp. 700707, Baltimore, MD, USA, July 2010.

[40] S. E. Houts, S. G. Dektor, and S. M. Rock, "A robust frame work for failure detection and recovery for terrain-relative navigation," in Proceedings of the Unmanned Untethered Submersible Technology, Portsmouth, NH, USA, September 2013.

[41] C. T. Francisico, Q. Joao, M. Pramod et al., "Robust particle filter formulations with application to terrain-aided navigation," International Journal of Adaptive Control and Signal Processing, vol. 31, pp. 608-651, 2017.

[42] D. Peng, T. Zhou, J. Folkesson, and C. Xu, "Robust particle filter based on Huber function for underwater terrain-aided navigation," IET Radar, Sonar \& Navigation, vol. 13, no. 11, pp. 1867-1875, 2019.

[43] Y. J. Liu, G. C. Zhang, R. Lu et al., "Study on Particle Filter Based Terrain Aided Navigation under the Ultra-low Resolution Map of the Arctic," in Proceedings of the 2020 39th Chinese Control Conference, Shenyang, China, July 2020.

[44] C. T. Francisco, P. Antonio, and M. Pramod, "A novel particle filter formulation with application to terrain-aided navigation," IFAC Proceedings Volumes, vol. 45, no. 5, pp. 132-139, 2012. 